\title{
NON-SMIRNOV DOMAINS
}

\author{
KNUT ØYMA
}

(Communicated by Clifford J. Earle, Jr.)

\begin{abstract}
If $\Omega$ is a Jordan domain, a small perturbation of the boundary gives a non-Smirnov domain.
\end{abstract}

Let $D$ be the open unit disc and $T$ its boundary. A conformal mapping $f(z)$ from $D$ onto a Jordan domain $\Omega$ extends to a homeomorphism between $\bar{D}$ and $\bar{\Omega}$ (the closures). This was proved by Osgood and Taylor and independently by Carathéodory (1913). The boundary of $\Omega, \partial \Omega$, is rectifiable if and only if $f^{\prime}(z) \in H^{1}$. See [1]. The $H^{p}$ spaces are treated in [2] and [4]. The length of $\partial \Omega$ is $|\partial \Omega|=\int_{0}^{2 \pi}\left|f^{\prime}\left(e^{i \theta}\right)\right| d \theta$. Because $f^{\prime}(z)$ belongs to $H^{1}$ we have $f^{\prime}(z)=S(z) F(z)$, where $S(z)$ is singular inner and $F(z)$ is outer. There is no Blaschke factor since $f(z)$ is univalent. If $S(z) \equiv 1$, then $\Omega$ is called a Smirnov domain. In such domains function theory inherits nice properties from the unit disc. See Chapter 10 of [2]. Non-Smirnov domains exist. An elegant proof is due to Duren, Shapiro, and Shields [3]. Keldysh and Lavrentiev gave the first example in 1937. A detailed version appears in the book of Privalov [6].

In this paper we will use the idea of Keldysh and Lavrentiev to show that the shape of such domains can roughly be prescribed. In particular the nonSmirnov domains are dense in the simply connected domains in the sense of Carathéodory.

Theorem. If $f(z)$ is univalent in $D, 0<r_{1}<r_{2}<1$, then there exists a nonSmirnov domain $\Delta$ such that $f\left(|z|<r_{1}\right) \subset \Delta \subset f\left(|z|<r_{2}\right)$. There exists a conformal mapping $\varphi(z)$ of $D$ onto $\Delta$ such that $\left|\varphi^{\prime}\left(e^{i \theta}\right)\right|=$ constant a.e.

The result has an interesting Brownian motion interpretation. Let $I$ be a measurable subset of $\partial \Delta$. Consider a Brownian motion starting at $\varphi(0)$. The probability for the first exit from $\Delta$ to take place on $I$ equals $|I| /|\partial \Delta|$. We need five lemmas.

Lemma 1 (Montel). Let $\Omega_{1} \subset \Omega_{2}$ be Jordan domains bounded by finitely many analytic arcs. Assume that $f_{i}(z)$ maps $\Omega_{i}$ conformally onto $D$ and that $f_{1}\left(z_{0}\right)$ $=f_{2}\left(z_{0}\right)$ for some $z_{0} \in \Omega_{1}$. If the open analytic arc $\Gamma$ is contained in $\partial \Omega_{1} \cap$ $\partial \Omega_{2}$, then $\left|f_{2}^{\prime}(z)\right| \geq\left|f_{1}^{\prime}(z)\right|$ for every $z \in \Gamma$.

Received by the editors June 9, 1990 and, in revised form, July 13, 1993.

1991 Mathematics Subject Classification. Primary 30C20; Secondary 30D55. 
A proof is found in [6], p. 28.

Let $I$ be a subarc of $T$ of length $2 \theta<\frac{\pi}{3}$. For $0 \leq \gamma \leq \frac{1}{2}, C_{\gamma}$ is the part of a circle through the endpoints of $I$ lying outside $D$ making the angle $\gamma \pi$ with $I$. $D_{\gamma}$ is the domain bounded by $(T \backslash I) \cup C_{\gamma}$. Let $h_{\gamma}(z)$ map $D_{\gamma}$ conformally onto $D$ such that $h_{\gamma}(0)=0$. The midpoint of $C_{\gamma}$ is $z_{0}$.

Lemma 2. The minimum of $\left|h_{\gamma}^{\prime}(z)\right|$ for $z \in C_{\gamma}$ is attained at $z_{0}$.

$$
\left|h_{\gamma}^{\prime}\left(z_{0}\right)\right|=\frac{2}{1+\gamma} \cdot \frac{\tan \frac{\theta}{2(1+\gamma)} \cos ^{2} \frac{\theta+\gamma \pi}{2}}{\sin \theta}
$$

A proof is in [6], p. 162.

We want to solve the equation $\left|h_{\gamma}^{\prime}\left(z_{0}\right)\right|=\mu<1$ with respect to $\gamma$.

Lemma 3. There exists a constant $c>0$ such that any solution $\gamma$ of the equation $\left|h_{\gamma}^{\prime}\left(z_{0}\right)\right|=\mu<1$ satisfies $\gamma \geq c(1-\mu)$.

Proof. By the mean value theorem we have

$$
\mu-1=\left|h_{\gamma}^{\prime}\left(z_{0}\right)\right|-\left|h_{0}^{\prime}\left(z_{0}\right)\right|=\left(\frac{\partial}{\partial \gamma}\left|h_{\gamma}^{\prime}\left(z_{0}\right)\right|\right)_{\gamma=t} \cdot \gamma
$$

for some number $t$ between 0 and $\gamma$.

Let $M=\operatorname{Max}\left\{\left|\frac{\partial}{\partial \gamma}\right| h_{\gamma}^{\prime}\left(z_{0}\right)||: 0 \leq \gamma \leq \frac{1}{2}, 2 \theta \leq \pi / 3\right\}$.

Then $1-\mu \leq M \cdot \gamma$. This proves the lemma with $c=M^{-1}$.

Lemma 4. There exists a strictly increasing function $g(\gamma)$ satisfying $g(0)=1$ and $\left|C_{\gamma}\right| \geq g(\gamma)|I|$.

The proof uses simple geometry and is omitted.

Lemma 5. Let $\varphi(z)$ be analytic in $D$. Assume that $\left|\varphi^{\prime}(z)\right|<1$ and that $\varphi^{\prime}(0) \geq$ $\delta>0$. If $E$ is a measurable subset of $T$ of length $s>0$, then $\int_{E}\left|\varphi^{\prime}\left(e^{i \theta}\right)\right| d \theta \geq$ $K=K(s, \delta)>0$.

Proof. Let $A=\left\{e^{i \theta}:\left|\varphi^{\prime}\left(e^{i \theta}\right)\right|<\varepsilon\right\}$ where $\varepsilon$ satisfies $\frac{2 \pi \log \delta}{\log \varepsilon}=\frac{s}{2}$. Since $\varphi^{\prime}(z) \in H^{1}$, we have $\log \delta \leq \frac{1}{2 \pi} \int_{T} \log \left|\varphi^{\prime}\left(e^{i \theta}\right)\right| d \theta \leq \frac{1}{2 \pi}|A| \log \varepsilon$. Therefore $|A| \leq \frac{2 \pi \log \delta}{\log \varepsilon}=\frac{s}{2}$. Hence $E(\varepsilon)=\left\{e^{i \theta} \in E:\left|\varphi^{\prime}\left(e^{i \theta}\right)\right| \geq \varepsilon\right\}$ satisfies $|E(\varepsilon)| \geq \frac{s}{2}$. This proves the lemma since $\int_{E}\left|\varphi^{\prime}\left(e^{i \theta}\right)\right| d \theta \geq \int_{E(\varepsilon)}\left|\varphi^{\prime}\left(e^{i \theta}\right)\right| d \theta \geq \frac{s}{2} \varepsilon=K(s, \delta)$.

We now prove the theorem. The proof is technical and the reader should make a drawing. We will construct Jordan domains $\Delta_{n}$ bounded by a finite number of analytic arcs such that $\Delta_{n} \subset \Delta_{n+1}$. The non-Smirnov domain will be the union of $\Delta_{n}$. By $\varphi_{n}(z)$ we mean the conformal mapping from $D$ onto $\Delta_{n}$ such that $\varphi_{n}(0)=0$ and $\varphi_{n}^{\prime}(0)=\operatorname{Re} \varphi_{n}^{\prime}(0)>0$.

Since $\Delta_{n}$ is bounded by finitely many analytic arcs, $\varphi_{n}(z)$ has a univalent continuation to a domain $D_{n}$ containing $D$. For $n>1, \partial D_{n}$ meets $T$ at a finite number of points. We define $\varphi_{n}\left(D_{n}\right)=\Omega_{n}$. These domains will satisfy $\Omega_{n} \supset \Omega_{n+1}$. For $n>1, \partial \Omega_{n}$ will meet $\partial \Delta_{n}$ at finitely many points. The inverse of $\varphi_{n}(z)$ is denoted $f_{n}(z)$. We construct the domains inductively.

We define $\Delta_{1}=f\left(|z|<r_{1}\right), D_{1}=\left\{|z|<\frac{r_{2}}{r_{1}}\right\}$ and $\Omega_{1}=f\left(|z|<r_{2}\right)$. We may assume that $f(0)=0$ and that $f^{\prime}(0)=\operatorname{Re} f^{\prime}(0)>0$. Then $\varphi_{1}(z)=f\left(r_{1} z\right)$ is properly normalized. By dilation we may assume that $\left|\varphi_{1}^{\prime}(z)\right| \leq 1$ for $z \in D$. 
Assume that $\Delta_{n}, D_{n}, \Omega_{n}$ and $\varphi_{n}(z)$ have been constructed and that $\left|\varphi_{n}^{\prime}(z)\right| \leq$ 1 for $z \in D$. Define $a_{n}$ and $A_{n}$ by

$$
\begin{gathered}
\int_{T}\left|\varphi_{n}^{\prime}\left(e^{i \theta}\right)\right| d \theta=\left|\partial \Delta_{n}\right|=2 \pi-a_{n}, \\
A_{n}=\left\{e^{i \theta}: e^{i \theta} \in D_{n},\left|\varphi_{n}^{\prime}\left(e^{i \theta}\right)\right|<1-\frac{a_{n}}{20}\right\} .
\end{gathered}
$$

This set satisfies $\int_{T \backslash A_{n}}\left|\varphi_{n}^{\prime}\left(e^{i \theta}\right)\right| \leq 2 \pi-a_{n}$. Therefore we have $\left(2 \pi-\left|A_{n}\right|\right)$ $\cdot\left(1-\frac{a_{n}}{20}\right) \leq 2 \pi-a_{n}$. This leads to $\left|A_{n}\right| \geq \frac{a_{n}}{2}$.

Let $\left\{I_{k}\right\}\left(=\left\{I_{k, n}\right\}\right)$ be finitely many disjoint closed subarcs of $A_{n}$. Each $I_{k}$ has length less than $\frac{\pi}{3}$ and is contained in a closed disc $O_{k}$ meeting the endpoints of $I_{k}$ at an angle of $\frac{\pi}{2}$. The following conditions are satisfied:

(i) $O_{k} \subset D_{n}$.

(ii) $\sum\left|I_{k}\right|>\frac{a_{n}}{4}$.

(iii) $\sup _{z \in O_{k}}\left|\varphi_{n}^{\prime}(z)\right|=\mu_{k}<1-\frac{a_{n}}{40}$.

(iv) diam $O_{k}<b_{n}$, where $b_{n}$ is a small number to be chosen later.

(v) $\left(\inf _{z \in O_{k}}\left|\phi_{n}^{\prime}(z)\right|\right) / \mu_{k}>r_{n}$, where $r_{n}$ is a number close to one to be chosen later.

Replace $I_{k}$ by a bubble as in Lemma 2 where $\gamma_{k}$ satisfies $\left|h_{\gamma_{k}}^{\prime}\left(z_{0}\right)\right|=\mu_{k}$. If no such $\gamma_{k}$ exists, let $\gamma_{k}=\frac{1}{2}$. Recall the definition of $D_{\gamma_{k}}$ and let $D_{n}^{*}=\bigcup D_{\gamma_{k}}$. Note that $\varphi_{n}(z)$ is univalent in $D_{n}^{*}$ and that $\varphi_{n}\left(D_{n}^{*}\right) \subset \Omega_{n}$. Let $h_{n}(z)$ map $D_{n}^{*}$ conformally onto $D$ such that $h_{n}(0)=0$ and $h_{n}^{\prime}(0)>0$. If $z \in \partial D_{n}^{*}$ and $|z|>1$, then $z \in C_{\gamma_{k}}$ for some $k$. Therefore $\left|h_{n}^{\prime}(z)\right| \geq\left|h_{\gamma_{k}}^{\prime}(z)\right| \geq\left|h_{\gamma_{k}}^{\prime}\left(z_{0}\right)\right| \geq$ $\mu_{k}$. The first inequality follows from Lemma 1 , the second from Lemma 2 , and the third inequality follows from the the definition of $\gamma_{k}$.

We define $\Delta_{n+1}=\varphi_{n}\left(D_{n}^{*}\right)$. Then $\Delta_{n} \subset \Delta_{n+1} \subset \Omega_{n}$. The boundary of $\Delta_{n+1}$ is rectifiable and consists of a finite number of analytic arcs. To prove that $\left|\varphi_{n+1}^{\prime}(z)\right| \leq 1$ for $z \in D$ it suffices to prove that $\left|f_{n+1}^{\prime}(z)\right| \geq 1$ a.e. on $\partial \Delta_{n+1}$. There are two cases. Assume that $z \in \partial \Delta_{n+1} \cap \partial \Delta_{n}$ and that both $f_{n}^{\prime}(z)$ and $f_{n+1}^{\prime}(z)$ exist. This excludes only a finite number of points on $\partial \Delta_{n+1} \cap \partial \Delta_{n}$. By Lemma 1 we have that $\left|f_{n+1}^{\prime}(z)\right| \geq\left|f_{n}^{\prime}(z)\right| \geq 1$. If $z \in \partial \Delta_{n+1} \backslash \partial \Delta_{n}$, then $f_{n}(z) \in C_{\gamma_{k}}$ for some $k$. Note that $f_{n+1}(z)=h_{n}\left(f_{n}(z)\right)$. Therefore $\left|f_{n+1}^{\prime}(z)\right|=\left|h_{n}^{\prime}\left(f_{n}(z)\right)\right| \cdot\left|f_{n}^{\prime}(z)\right| \geq \mu_{k} \cdot \frac{1}{\left|\varphi_{n}^{\prime}\left(f_{n}(z)\right)\right|} \geq \mu_{k} \cdot \frac{1}{\mu_{k}}=1$ by (iii). This proves that $\left|\varphi_{n+1}^{\prime}(z)\right| \leq 1$ in $D$ and that $\left|\partial \Delta_{n+1}\right| \leq 2 \pi$. Recall that $\Delta_{n+1} \subset \Omega_{n}$ and that $\partial \Omega_{n}$ meets $\partial \Delta_{n+1}$ at a finite number of points. As before $\varphi_{n+1}(z)$ has a univalent continuation to a domain $D_{n+1}$ containing $D$ such that $\left(\partial D_{n+1} \cap T\right)$ is finite. If necessary we decrease $D_{n+1}$ by choosing $\partial D_{n+1}$ close to $T$ to obtain:

(1) $\varphi_{n+1}\left(D_{n+1}\right)=\Omega_{n+1} \subset \Omega_{n}$.

(2) Any Jordan curve $\Gamma$ in $\bar{\Omega}_{n+1}$ surrounding $\Delta_{n+1}$ must satisfy $|\Gamma| \geq$ $\left|\partial \Delta_{n+1}\right|-\frac{1}{n}$.

We now prove that $\left|\partial \Delta_{n}\right|$ is increasing. It follows from (v) that

$$
\frac{\int_{C_{\gamma_{k}}}\left|\varphi_{n}^{\prime}(z)\right| d s}{\int_{I_{k}}\left|\varphi_{n}^{\prime}(z)\right| d s} \geq \frac{\left|C_{\gamma_{k}}\right|}{\left|I_{k}\right|} r_{n} .
$$


Note that $\gamma_{k} \geq c\left(1-\mu_{k}\right) \geq c \frac{a_{n}}{40}$ by Lemma 3 and (iii). By Lemma $4 \frac{\left|C_{\gamma_{1}}\right|}{\left|I_{k}\right|} \geq$ $g\left(c \frac{a_{n}}{40}\right)=\zeta^{\prime}>1$. Combining these inequalities we obtain

$$
\int_{C_{\gamma_{k}}}\left|\varphi_{n}^{\prime}(z)\right| d s \geq \zeta^{\prime} r_{n} \int_{I_{k}}\left|\varphi_{n}^{\prime}(z)\right| d s
$$

We now choose $r_{n}$ such that $\zeta^{\prime} r_{n}=\frac{1+\zeta^{\prime}}{2}=\zeta$. Consequently

$$
\begin{aligned}
\left|\partial \Delta_{n+1}\right|-\left|\partial \Delta_{n}\right| & =\sum_{k}\left(\int_{C_{\gamma_{k}}}\left|\varphi_{n}^{\prime}(z)\right| d s-\int_{I_{k}}\left|\varphi_{n}^{\prime}(z)\right| d s\right) \\
& \geq(\zeta-1) \sum_{k} \int_{I_{k}}\left|\varphi_{n}^{\prime}(z)\right| d s=\frac{g\left(c \frac{a_{n}}{40}\right)-1}{2} \int_{\cup I_{k}}\left|\varphi_{n}^{\prime}(z)\right| d s
\end{aligned}
$$

this proves that $\left|\partial \Delta_{n}\right|$ is increasing.

Since $\left|\partial \Delta_{n}\right| \leq 2 \pi$ it follows that $\lim _{n \rightarrow \infty}\left|\partial \Delta_{n}\right|$ exists. Assume that this limit equals $2 \pi-a$ where $a>0$. Then $a_{n}>a$ for all $n$. Subordination, a variant of Schwarz' lemma, proves that $\varphi_{n}^{\prime}(0) \geq \varphi_{1}^{\prime}(0)=\delta>0$. For all $n$ we have that $\left|\bigcup I_{k}\right|>\frac{a}{40}$. Apply Lemma 5 and $(*)$ :

$$
\begin{aligned}
\left|\partial \Delta_{n+1}\right|-\left|\partial \Delta_{n}\right| & \geq \frac{g\left(c \frac{a}{40}\right)-1}{2} \int_{\cup I_{k}}\left|\varphi_{n}^{\prime}(z)\right| d s \\
& \geq \frac{g\left(c \frac{a}{40}\right)-1}{2} K\left(\frac{a}{40}, \delta\right)>0 .
\end{aligned}
$$

This is a contradiction, hence $\lim \left|\partial \Delta_{n}\right|=2 \pi$. Let $\Delta=\bigcup \Delta_{n}$. To prove that $\Delta$ is a Jordan domain recall that $\Delta_{n}=\varphi_{n}(D)$ and that $\Delta_{n+1}=\varphi_{n}\left(D_{n}^{*}\right)$. By construction every point of $D_{n}^{*}$ can be connected to a point in $D$ by a line segment of length less than $b_{n}$. See (iv). Since $\left|\varphi_{n}^{\prime}(z)\right|<1$ everywhere in $D_{n}^{*}$, every point in $\Delta_{n+1}$ can be connected to a point in $\Delta_{n}$ by a curve of length less than $b_{n}$. By induction every point in $\Delta$ can be connected to a point in $\Delta_{N}$ by a curve of length less than $\sum_{n \geq N} b_{n}$. A domain is a Jordan domain if and only if for every $\varepsilon>0$ there exists a $\delta>0$ such that any two points closer together than $\delta$ lie in a connected subset of diameter less than $\varepsilon$. We may assume that $\delta<\varepsilon$. For every $N, \Delta_{N}$ is a Jordan domain. If $\varepsilon_{N}=\frac{1}{2^{N}}$. there exists $\delta_{N}$ corresponding to $\varepsilon_{N}$ that works for $\Delta_{N}$. Choose the numbers $b_{n}$ in (iv) such that $\sum_{n \geq N} b_{n}<\frac{1}{3} \delta_{N}$. Let $z_{1}$ and $z_{2}$ be two points in $\Delta$ such that $\left|z_{1}-z_{2}\right|<\frac{\delta_{N}}{3}$. For $i=1,2$ choose curves $K_{i}$ of length less than $\frac{\delta_{N}}{3}$ connecting $z_{i}$ with $w_{i} \in \Delta_{N}$. Then $\left|w_{1}-w_{2}\right|<\delta_{N}$. Choose a connected set $E \subset \Delta_{N}$ of diameter less than $\varepsilon_{N}$ such that $w_{i} \in E$. The set $\left(E \cup K_{1} \cup K_{2}\right)$ is connected, has diameter less than $2 \varepsilon_{N}$, and contains $z_{i}$. Hence $\Delta$ is a Jordan domain.

Let $\varphi(z)$ be the conformal mapping of $D$ onto $\Delta$ normalized by $\varphi(0)=0$ and $\varphi^{\prime}(0)=\operatorname{Re} \varphi^{\prime}(0)>0$. Since $\varphi_{n}^{\prime}(z) \rightarrow \varphi^{\prime}(z)$ uniformly on compact sets, we have that $|\partial \Delta| \leq 2 \pi$. Condition (2) shows that $|\partial \Delta| \geq\left|\partial \Delta_{n+1}\right|-\frac{1}{n}$. Therefore $|\partial \Delta|=2 \pi$ and the proof is complete unless $\varphi^{\prime}(z) \equiv 1$. By the dilatation argument in the beginning of the proof we may assume that $\Omega_{1} \subset\left\{|z|<\frac{1}{2}\right\}$. Since $\Delta \subset \Omega_{1}$, this cannot be the case. 


\section{REFERENCES}

1. E. F. Collingwood and A. J. Lohwater, The theory of cluster sets, Cambridge Univ. Press, Cambridge and New York, 1966.

2. P. Duren, Theory of $H^{p}$ spaces, Academic Press, New York, 1970.

3. P. Duren, H. S. Shapiro, and A. Shields, Singular measures and domains not of Smirnov type, Duke Math. J. 33 (1966), 247-254.

4. J. Garnett, Bounded analytic functions, Academic Press, New York, 1981.

5. M. W. Keldysh and M. A. Lavrentiev, Sur la représentation conforme des domaines limités par des courbes rectifiables, Ann. Sci. École Norm. Sup. 54 (1937), 1-38.

6. I. I. Privalov, Randeigenschaften analytischer Funktionen, Deutscher Verlag, Berlin, 1956.

Department of Mathematics, Agder College, 4604 Kristiansand, Norway 